\title{
Simulation of Automated Machine Tool Systems
}

\author{
Elena Dumler ${ }^{1}$, Roza Vakhitova $^{2}$, Diana Saracheva ${ }^{2}$, Tatyana Makarova ${ }^{2}$ \\ ${ }^{1}$ Ufa State Petroleum Technical University, RU-450064, Ufa, Republic of Bashkortostan, Russia \\ ${ }^{2}$ Almetyevsk State Oil Institute, RU-423450, Tatarstan, Almetyevsk, Russia
}

\begin{abstract}
A method of multi-nomenclature production in terms of technical and economic indicators was considered. An integrated design system that is able to assess the real relationship in the system "partmachine-transport device-device-tool" was proposed, and arelationship between the elements of an automated machine tool system was examined. The structure of an automated machine tool system, proposed in the form of a mixed graph, represented by sets described by nonlinear equations, was considered. The definitions of the sets of elements for an automated machine tool system that characterize the properties of technological objects were formulated. The main objects of design in the technological project of an automated machine system of multi-nomenclature production were determined and a graphical interpretation of the automated machine tool system was presented. It was revealed that technological equipment, the main technological machines, cutting and auxiliary tools and vehicles adequately describe manufacturing process for group of parts and are the main objects of designing technological projects for an automated machine system of multi-product manufacturing. The relationships between the elements indicated in the graph allow us to formalize and to establish the relationship between the individual components of an automated machine system, indicating in this way the need to develop a formal methodology for synthesizing the structure of an automated machine system.
\end{abstract}

\section{Introduction}

The current state of robotics, machine tools, informatics and other related industries, the features of multinomenclature production and the level of automation of metalworking at industrial enterprises have made it possible to formulate and to realize the concept of an automated machine tool system of multi-nomenclature production (ACC MNP) [1, 2].

Recently, the structure of the design stages of a multi-nomenclature production, which is based on the principles of group organization, has been developed [3 - 5]. The basic system-structural approaches to design were also developed. The relationship between the elements of the ACC such as the main technological equipment, auxiliary and cutting tools, vehicles and technological equipment at the level of the technological stratum were investigated; however, this investigation was not deep enough to fulfil all practical needs and, thus, need further refinement.

The production efficiency depends, to a large extent on the rational choice of equipment. As for now, no efficient methods of separating, from a huge total set of equipment, a set of machine tools, which are most expedient in terms of technical and economic indicators for processing parts of a certain nomenclature, have been proposed for [6, 7].

It is clear that a thorough investigation of the relationship between the elements of the ACC is possible only when using an integrated design system capable of assessing the real relationships in the system "part machine - transport device - device - tool" [8, 9].

\section{The structure of the automated machine tool system}

The structure of the automated machine tool system can be represented as an arbitrary mixed graph:

$$
G=(Z, U, P),
$$

where $Z$ is a plurality of elements of an automated machine tool system;

$U$ - many relationships between the elements of the automatedmachine system,

$P$ - is a triple predicate, called an incident.

The set $\{Z\}$ of elements of the automated machine tool system defines a subset of units of vehicles, main process equipment, devices and warehouses for their maintenance, measuring and cutting tools, technological equipment, instrumentation system, control and measuring devices and functional support aids.

Set $\{U\}$, which is determined by the set of information and material relationships that arise between elements of the automated machine tool system during its operation.

The graph $\mathrm{G}$ corresponds to the conditions in which: the incident $\mathrm{P}$ is set in the ordered triples of elementsz, $y$, $u$, where $y \in\{Z\}$, a $u \in\{U\}$; 


$$
\begin{gathered}
P\left(z^{\prime}, u, y^{\prime}\right) \rightarrow\left[\left(z=z^{\prime} \wedge y=y^{\prime}\right) \vee\left(z=y^{\prime} \wedge y=z^{\prime}\right)\right] \\
(\forall u \in U)(\forall z, y \in Z)\left[P(z, u, y) \wedge\left(\forall z^{\prime}, y^{\prime} \in Z\right)\right]
\end{gathered}
$$

The properties of an arbitrary mixed graph are such that for any value and $u \in\{U\}$ one of three expressions is true:

$$
\begin{gathered}
(\forall z, y \in Z)[P(z, u, y) \rightarrow \bar{P}(z, u, y)], \\
(\exists z, y \in Z)[P(z, u, y) \wedge P(y, u, z) \wedge(z \neq y)], \\
(\exists z \in Z)[P(z, u, z)],
\end{gathered}
$$

Depending on the fairness of the statement, the set $\{U\}$ is divided in pairs into three disjoint subsets $\{\vec{U}, \tilde{U}, \dot{U}\}$, which are respectively called arcs, links and loops.

Thus, the graph describing the structure of the automated machine tool system $G=(Z, U, P)$ between its two vertices contains links and arcs running in the same direction, and has several loops at one vertex, i.e. $\vec{U} \neq 0, \tilde{U} \neq 0, \stackrel{\circ}{U} \neq 0$.

As an example, figure 1 shows a graphical interpretation of an automated machine tool system, which includes:

$\mathrm{Z}_{11}$-warehouse of blanks, finished parts;

$\mathrm{Z}_{12}, \mathrm{Z}_{\mathrm{I3}}$ - stores;

$\mathrm{Z}_{21}, \mathrm{Z}_{22}$ - transport robots intended for delivery tools and parts for machines;

metalworking machines:

$\mathrm{Z}_{31}, \mathrm{Z}_{32}$-two turning, $\mathrm{Z}_{33}, \mathrm{Z}_{34}$-two milling;

$\mathrm{Z}_{5}$-the processing center;

$\mathrm{Z}_{36}$-grinder;

$\mathrm{Z}_{41}, \mathrm{Z}_{42}-$ control and measuring equipment;

$\mathrm{Z}_{51}$-tool storage room;

$\mathrm{Z}_{61}$-units of an overload of details;

$\mathrm{Z}_{71}$-management systems.

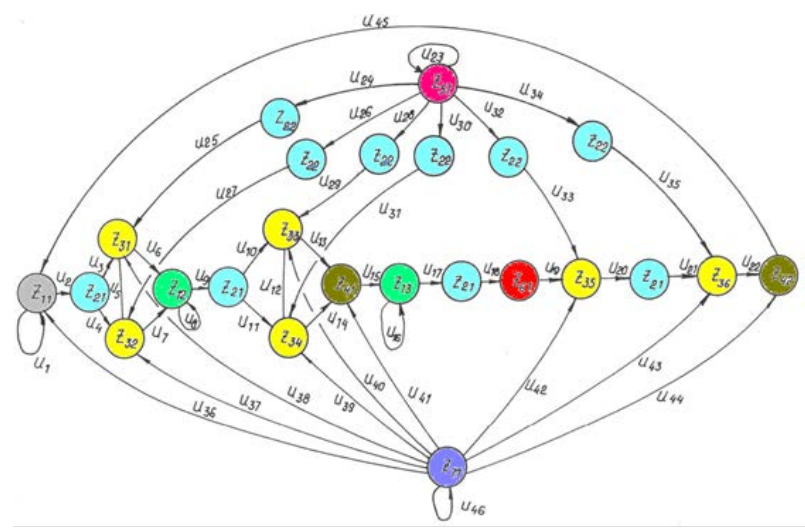

Fig. 1.Graphical interpretation of the structure of the machine tool system

\section{Mathematical model of the structure of an automated machine tool system}

For the automated machine system under investigation, we will build an incident $P$ and a set of $\{Z\},\{U\}$ :

$$
\begin{aligned}
& Z=\left\{Z_{11}, Z_{12}, Z_{12}, Z_{210}, Z_{22}, Z_{91}, Z_{22}, Z_{21}, Z_{24}, Z_{95}, Z_{96}\right. \\
& \left.Z_{41_{1}}, Z_{42}, Z_{51}, Z_{61}, Z_{71}\right\}
\end{aligned}
$$

Indicators that satisfy one of the properties for the graph with the incident P described by formulae (2), (3), (4) are true, and the indicators on all other triples of elements are false. In the mixed graph $G=(Z, U, P)$, the existing set of edges $U$ is divided into a subset of arcs:

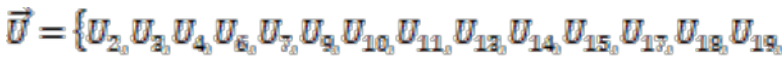

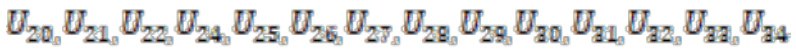

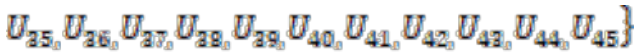

where $U=\left\{U_{1}, U_{8}, U_{16}, U_{23}, U_{46}\right\}$ is a subset of loops; $U=\left\{U_{5}, U_{12}\right\}$ is a subset of links.

Subsets $\vec{U}, \tilde{U}, \stackrel{\cup}{U}$ determine technological capabilities of warehouses and control system and characteristics of information and material flows of processing equipment in automated machine-tool system.

From the formal model of the automated machine tool system represented by the arbitrary mixed graph $G$ $=(Z, U, P)$, we distinguish the functional medium, which is defined by the expression:

$$
G=\left(X, O, T, I, D_{2}, I^{\prime}, E_{i}\right) i=1,5
$$

where $X=\left\{a_{1}, a_{2}, \ldots, a_{x}, a_{X}\right\}$ - a set of the main technological equipment units;

$O=\left\{p r_{1}, p r_{2}, \ldots, p r_{o}, p r_{O}\right\}-$ a plurality of process tooling units (different accessories);

$T=\left\{b_{1}, b_{2}, \ldots, b_{t}, b_{T}\right\}-$ a plurality of vehicles for tools and parts;

$I=\left\{\mathrm{in}_{1}, i \mathrm{in}_{2}, \ldots, i \mathrm{in}_{i}, i \mathrm{n}_{I}\right\}-$ a plurality of auxiliary and cutting tools;

$D_{g}=\left\{d_{1}, d_{2}, \ldots, d_{M}\right\}, 2 \in\{\Gamma\} \quad$ - Multiple Group Processing Technologies parts, as well as design and technological characteristics;

$I^{\prime}=\left\{i z_{1}, i z_{2}, \ldots, i z_{i^{\prime}}, i z_{I^{\prime}}\right\}-$ a plurality of monitoring and measuring machines and tools;

$E_{i}, i^{\prime} 1$,5receives the following values:

$E_{1}=\left\{e_{1}^{1}, e_{2}^{1}, \ldots, e_{E_{1}}^{1}\right\}$ - multiple items of warehouse equipment;

$E_{2}=\left\{e_{1}^{2}, e_{2}^{2}, \ldots, e_{E_{2}}^{2}\right\}-$ a plurality of control system elements;

$E_{3}=\left\{e_{1}^{3}, e_{2}^{3}, \ldots, e_{E_{3}}^{3}\right\}-$ a many pieces of equipment for the movement of cutting fluids;

$E_{4}=\left\{e_{1}^{4}, e_{2}^{4}, \ldots, e_{E_{4}}^{4}\right\}-$ Multiple units of machinery and equipment of the industrial waste management system;

$E_{5}=\left\{e_{1}^{5}, e_{2}^{5}, \ldots, e_{E_{5}}^{5}\right\} \quad-$ multiple elements of information flows in the ACC.

The identity of the service purpose, structural and technological elements, manufacturing material, technological process, machinery and equipment, tools and technological equipment used in production are characterized by the groups of parts $D_{g}, \quad z \in\{\Gamma\}$, and therefore, the expression is valid for them: 


$$
D_{g}=A_{j=1}^{M_{1}} d_{i}=\left\{\begin{array}{c}
d_{0}=\left\{\beta_{1_{1}} \beta_{2, \ldots,} \beta_{G}\right\} \\
p=\left\{\gamma_{1} \gamma_{2}, \ldots, \gamma_{\mu}\right\} \\
S_{d_{1}}=G(N, E)
\end{array}\right\}
$$

where $M^{\prime}$ - is the number of parts in the group; $i \in\left\{N^{\prime}\right\}$,constituting the group;

$S_{d i}$-the structure of the set of parts dj represented by the mixed graph $G(N, E)$, in which the surfaces correspond to vertices, and the relations and operations to edges, characterize the mutual relationship of the surfaces described by the expression:

$$
E=\left(R_{p_{i}}, F_{p_{i}}\right), i \in\{N\}
$$

$d_{0}$-General information about part group $\mathrm{Dg}$, $\imath \in\{\Gamma\}_{\text {including design and process codes, workpiece }}$ types, material, and so on.

In different groups $\mathrm{Dg}$, the description parameters $d_{0}$ and $p_{i}$ and the set can be changed, however, all these changes do not affect the conventional design methodology and are quantitative.

According to definition 1 for formula (5), the expression:

$$
\{\mathrm{X}\} \wedge\{0\} \wedge\{\mathrm{T}\} \wedge\{\mathrm{I}\} \wedge\left\{\mathrm{I}^{\prime}\right\} \wedge\left\{\mathrm{E}_{i}\right\}=\{\mathrm{Z}\}, \quad i \in 1,5,
$$

The main process machines and equipment, technological equipment, cutting and auxiliary tools, vehicles, all of the above, ultimately provides the technological process of processing a group of parts and is the main design objects when creating the technological design of an automated machine system of multi-item production.

Thus, in the process design of an automated machine tool system of multi-nomenclature production, the graph described by the expression:

$$
G=(X, O, T, I, D),
$$

The relationships between the sets $\{X\},\{O\},\{T\},\{I\}$, $\{D\}$, i.e. between the elements of the model presented in formula (8) create analytical relationships and are available for mathematical interpretation, which makes it possible to move to formalized regulation when designing elements of an automated machine tool system of multi-nomenclature production and are the main subject of system research.

Relationships between processing objects represented by processing means and set $\{D\}$ cover sets $\{X\},\{O\}$, $\{T\},\{I\}$ and form relationships arising between model elements (8).

Relationships are based on some properties of process objects of the automated machine tool system, such as equivalence, compatibility, following and interaction.

We formulate definitions characterizing the properties of technological objects.

Equivalence is defined as the equality of technological capabilities or properties of technical objects of an automated machine tool system (definition 3).

This attitude is indicated by a sign $\equiv$.

Let us present this definition as an expression in the form of symbols:

$$
\underset{X}{\forall a_{x}} \forall a_{x} a_{S} \exists S_{x} \exists S_{S} \ni P\left\{\left(a_{x}\left[S_{x}\right]=a_{q} \mid S_{q}\right) \wedge(x \neq q)\right\} \Rightarrow\left\lfloor a_{x}=a_{q}\right\rfloor(9)
$$

where $\{S\}$ - is a set of properties.

Process objects can be shared, provided they are defined by the same properties.

This attitude is indicated by a sign $\leftrightarrow$.

Let us present this definition in the form of characters:

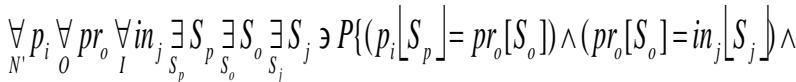

$$
\begin{aligned}
& \left.\wedge\left(p_{i}\left[S_{p}\right]=i n_{j}\left[S_{j}\right]\right)\right\} \Rightarrow\left[\left(p_{i} \leftrightarrow p r_{o}\right) \wedge\left(p r_{o} \leftrightarrow i n_{j}\right) \wedge\left(p_{i} \leftrightarrow i n_{j}\right)\right], \\
& i \in\left\{N^{\prime}\right\}, o \in\{O\}, j \in\{I\}
\end{aligned}
$$

wherein $S_{p}, S_{o}, S_{j}$ are a plurality of properties of machined surfaces, devices, tools, respectively.

Any element of the automated machine tool system is associated with another compatibility ratio (10).

If the structural, process and organizationalfunctional properties of a process object are considered in a certain sequence, then these two objects in the production process follow each other.

This attitude is indicated by a sign $\mapsto$.

Let's represent this relationship as symbols:

$$
\begin{gathered}
\underset{X}{\exists} a_{x} \exists a_{x} a_{T} b_{t} \ni P\left[P\left(a_{x}, \vec{U}, a_{q}\right) \wedge \bar{P}\left(a_{q}, \vec{U}, a_{x}\right) \wedge P\left(b_{t}, \vec{U}, a_{x}\right) \wedge \bar{P}\left(b_{t}, \vec{U}, a_{q}\right)\right] \Rightarrow \\
\Rightarrow\left[\left(b_{t} \mapsto a_{x}\right) \wedge\left(a_{x} \mapsto a_{q}\right)\right]
\end{gathered}
$$

Obviously (11), all elements of the automated machine tool system can be arranged according to the properties (2) to (4) and in the processing sequence.

If process objects work together, have the same properties and implement any functions or actions, then they interact.

The interaction relationship is indicated by the sign $><$.

Let's represent this relationship with characters:

$$
\begin{aligned}
& \underset{M}{\forall} d_{k} \underset{N^{\prime}}{\exists} p_{i} \in \underset{T}{\exists} b_{t} \underset{X}{\exists} a_{x} \underset{O}{\exists} \operatorname{pr}_{o} \underset{I}{\exists i n_{j}} \underset{S_{d}}{\exists} S_{d_{k}} \underset{S_{p}}{\exists} S_{p} \underset{S_{t}}{\exists} S_{t} \underset{S_{x}}{\exists} S_{x} \underset{S_{o}}{\exists} S_{o} \underset{S_{j}}{\exists} S_{j} \ni \\
& \quad \ni P\left\{P\left(b_{t}, \vec{U}, a_{x}\right) \wedge\left(d_{k}\left[S_{d}\right]=b_{t}\left[S_{t}\right]\right) \wedge\left[a_{x}\left[S_{x}\right]\right]=\right. \\
& \left.=p_{i}\left[S_{p}\right]=p r_{0}\left[S_{0}\right]=i n_{j}\left[S_{j}\right]\right\} \Rightarrow \\
& \Rightarrow\left[\left(b_{t}><a_{X}\right) \wedge\left(b_{t}><d_{k}\right)\right] \wedge\left[a_{x}><p_{i}><p r_{0}><i n_{j}\right], i \in\left\{1, N^{\prime}\right\}
\end{aligned}
$$

When several elements of the automated machine tool system are connected to each other by an interaction relationship in pairs, it is possible that elements not included in each pair may interact with each other, i.e.

$$
P\left[\left(b_{t}><a_{x}\right) \wedge\left(a_{x}><p_{i}\right) \wedge\left(p_{i}><p r_{0}\right)\right] \Rightarrow\left(b_{t}><p r_{0}\right)\left(a_{x}><p r_{0}\right) .
$$

Therefore, if the expression (12) is true, then it can be confidently considered that any of the elements of the 
ACC can be related by the relation of interaction with any other element thereof.

Figure 2 shows a graph constructed in accordance with the definitions of the relationships between the sets of the process environment of the automated machine system.

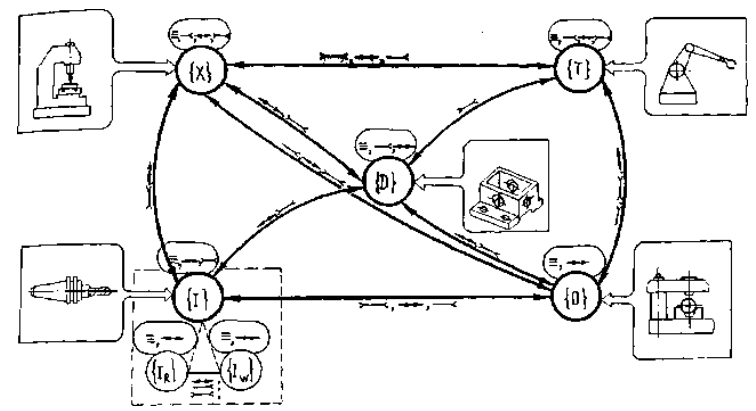

Fig. 2.Graph of relationships of process medium sets of automated system.

On the graph, the relationships between elements of the same or different sets are highlighted.

\section{Conclusion}

The relationships between the elements indicated on the graph allow formalizing and establishing the relationship between individual components of the automated machine tool system, indicating the need to develop a formal methodology for synthesizing the structure of the automated machine tool system.

The main machines and equipment, cutting and auxiliary tools, vehicles and other relevant components fully describe and provide the functionality of the technological process of manufacturing a group of parts and are the main design objects when developing a technological design of an automated machine system of a multi-nomenclature production.

\section{References}

1. S.N. Sharapov, A.V. Zaitsev, Directory, Engineering Journal with Annex, 6(267) (2019) ISSN: 0203-347X

2. S.G. Mitin, I.A. Razmanov, P.Yu. Bochkaryov, Directory, Engineering Journal with Annex, 5(242) (2017)

ISSN: 0203-347X

3. I.I. Kovtun, G.S. Romanenko, Informatization and Communication, 1 (2020) [in Russia] ISSN: 2078-8320

4. E.G. Krylov, S.V. Shurkhovetskiy, D.T. Arstangaliev, E.V. Reznikova, Magistracy Bulletin, 6(93) (2019) [in Russia] ISSN 2223-4047

5. S.S. Sergeev, L.G. Bokova, Promising methods for automating the creation of technological operations in multi-product production (Collection of articles of the XXV international scientific and practical conference, 2019)
6. R.I. Vakhitova, E.B. Dumler, F.Z., Bulyukova, E.F. Zakharova, Increasing the efficiency of diversified production based on modeling automated technological equipment: monograph (Almetyevsk, AGNI, 2019) ISBN: 978-5-94454-039-3

7. E.B. Dumler Voronezh State Technical University Bulletin, 3(5) (2007) ISSN 17296501

8. T.G. Devzheeva, New design principles in automated systems. Thermophysical and technological aspects of increasing the efficiency of machine-building production (Works of the IV International Scientific and Technical Conference (Reznikov readings), 2015)

ISBN: 978-5-8259-0872-4

9. I.P. Sitdikova, E.B. Dumler, R.I. Vakhitova, N.V. Abdulkina, Design of automated machine tools for multi-item production (Achievements, problems and prospects for the development of the oil and gas industry. Proceedings of the IV International Scientific and Practical Conference, 2019). ISBN: 978-5-94454-045-4 